\title{
Seed particle formation for silicate dust condensation by SiO nucleation ${ }^{\star}$
}

\author{
H.-P. Gail ${ }^{1}$, S. Wetzel ${ }^{2}$, A. Pucci ${ }^{2}$, and A. Tamanai ${ }^{2}$ \\ 1 Universität Heidelberg, Zentrum für Astronomie, Institut für Theoretische Astrophysik, Albert-Ueberle-Str. 2, 69120 Heidelberg, \\ Germany \\ e-mail: gail@uni-heidelberg.de \\ 2 Universität Heidelberg, Kirchhoff-Institut für Physik, Im Neuenheimer Feld 227, 69120 Heidelberg, Germany
}

Received 30 April 2013 / Accepted 16 May 2013

\begin{abstract}
Context. Dust formation in stellar outflows is initiated by the formation of some seed particles that form the growth centres for macroscopic dust grains. The nature of the seed particles for silicate dust in stellar outflows with an oxygen-rich element mixture is still an open question. Clustering of the abundant $\mathrm{SiO}$ molecules has been discussed several times as a possible mechanism and investigated both theoretically and by laboratory experiments. The initial results seemed to indicate, however, that condensation temperatures obtained by model calculations based on this mechanism are significant lower than what is really observed, which renders $\mathrm{SiO}$ nucleation unlikely.

Aims. This negative result strongly rests on experimental data on the vapour pressure of $\mathrm{SiO}$. The case for $\mathrm{SiO}$ nucleation may be not as bad as it previously seemed and needs to be discussed again because new determinations of the vapour pressure of $\mathrm{SiO}$ molecules over solid $\mathrm{SiO}$ have shown the older data on $\mathrm{SiO}$ vapour pressure to be seriously in error. Here we aim to check again the possibility that $\mathrm{SiO}$ nucleation triggers the cosmic silicate dust formation in light of improved new data.

Methods. First we present results of our measurements of vapour pressure of solid SiO. Second, we use the improved vapour pressure data to recalibrate existing experimental data on $\mathrm{SiO}$ nucleation from the literature. Third, we use the recalibrated data on $\mathrm{SiO}$ nucleation in a simple model program for dust-driven winds to determine the condensation temperature of silicate in stellar outflows from AGB stars.

Results. Our measurements extend the temperature range of measurements for the vapour pressure to lower temperatures and pressures than ever before. This improves the reliability of the required extrapolation from the temperature range where laboratory data can be obtained to the temperature range where circumstellar dust condensation is observed. We determine an analytical fit for the nucleation rate of $\mathrm{SiO}$ from recalibrated literature data and show that the onset of nucleation under circumstellar conditions commences at a higher temperature than was previously found. This brings calculated condensation temperatures of silicate dust much closer to the observed condensation temperatures derived from analysis of infrared spectra from dust-enshrouded M stars. Calculated condensation temperatures are still by about $100 \mathrm{~K}$ lower than observed ones, but this may be due to the greenhouse effect of silicate dust temperatures, which is not considered in our model calculation.

Conclusions. The assumption that the onset of dust formation in late-type stars with oxygen-rich element mixtures is triggered by the cluster formation of $\mathrm{SiO}$ is compatible with dust condensation temperatures derived from infrared observations.
\end{abstract}

Key words. stars: AGB and post-AGB - stars: winds, outflows - circumstellar matter - astrochemistry - methods: laboratory

\section{Introduction}

Outflow of matter in the stellar winds of highly evolved stars is observed to be accompanied by copious dust formation. As long as the element mixture in the outflow remains oxygenrich, the dominating dust components are magnesium-ironsilicates (see Molster et al. 2010, for a review of observations). Since the outflowing gas from the star is free of condensation seeds, the first step in the sequence of events ultimately ending with the growth of silicate dust grains in the cooling outflow has to be the formation of seed particles. Because $\mathrm{SiO}$ is one of the most abundant of all the gas-phase species of refractory elements that could be involved in the process, it has been discussed several times whether it is cluster formation by this species that is

\footnotetext{
* Appendix A is available in electronic form at http://www. aanda.org
}

at the basis of all the processes (Donn et al. 1981; Nuth \& Donn 1982, 1983; Gail \& Sedlmayr 1986, 1998a,b, Ali \& Castleman 2005; Nuth \& Ferguson 2006; Reber et al. 2006; Paquette et al. 2011; Goumans \& Bromley 2012) and that this ultimately leads to the condensation of silicate dust particles.

The reason that formation of large complexes of $\mathrm{SiO}$ molecules or of solid $\mathrm{SiO}$ is thought to be an important intermediate step in the formation of silicate dust is as follows: there are no molecular species with a composition that corresponds to the chemical formula of silicate compounds as free gas-phase species (at least not in detectable amounts). It is necessary, therefore, that the initial stages of the condensation of silicates involve some different kind of material. It has been thought since the early 1980 s that condensation of $\mathrm{SiO}$ is the most obvious initial step in silicate formation (e.g. Nuth \& Donn 1982; Gail \& Sedlmayr 1986) because of the high abundance of $\mathrm{SiO}$ molecules in the gas phase in oxygen-rich environments 
and, in particular, because a rather stable condensed phase exists with chemical composition $\mathrm{SiO}$. It is assumed that initially silicon monoxide particles form which then serve as seed particles for growth of silicates. There is even the possibility that in a number of cases the silicon-monoxide seed particles finally grow to a dust species of their own (Wetzel et al. 2013).

Some dedicated laboratory experiments have been performed to study the nucleation and condensation of $\mathrm{SiO}$ (Nuth $\&$ Donn 1982). It turned out that the results suggest an onset of condensation at much too low a temperature to be compatible with observed condensation temperatures in circumstellar dust shells. Theoretical calculations (Gail \& Sedlmayr 1986) based on the results of Nuth \& Donn (1982) show that nucleation of $\mathrm{SiO}$ should not commence before temperature has dropped below $\approx 600 \mathrm{~K}$, while observed condensation temperatures in circumstellar dust shells are typically of the order of $900 \mathrm{~K}$ or sometimes even higher. Alternatives to seed formation by $\mathrm{SiO}$ are nucleation of oxides of, e.g., Al or Ti (Gail \& Sedlmayr 1998a,b; Jeong et al. 1998). Such mechanisms, however, raise serious problems. Because of the low elemental abundances of $\mathrm{Al}$ or $\mathrm{Ti}$, seed particle formation at mass-loss rates as low as $10^{-7} M_{\odot} a^{-1}$ would occur at a prohibitively low rate, while it is observed that even at such low mass-loss rates at least some dust can be formed. The proposed alternatives to $\mathrm{SiO}$ nucleation were therefore not successful.

It therefore seems worthwhile to investigate the case of $\mathrm{SiO}$ nucleation again and to look for possibilities for circumventing the difficulties with this mechanism that in the past led to a rejection of the $\mathrm{SiO}$-nucleation hypothesis. Nuth \& Ferguson (2006) and Ferguson \& Nuth (2008) addressed the problem of the vapour pressure of $\mathrm{SiO}$ and found this to be considerably lower than previous measurements (Schick 1960) had suggested. This would allow condensation of $\mathrm{SiO}$ to occur at higher temperatures than previously thought. They argued on this basis (Nuth \& Ferguson 2006) that $\mathrm{SiO}$ could be the first species to nucleate in the outflow from a star with oxygen-rich element mixture if the effects of non-TE level population of $\mathrm{SiO}$ molecules are also accounted for (Nuth \& Donn 1981). But even then condensation temperatures do not fit observations very well.

We also performed a new determination of the vapour pressure of $\mathrm{SiO}$ (Wetzel et al. 2012) and used the result to re-evaluate the old experimental results of Nuth \& Donn (1982) on SiO nucleation using our new data for $\mathrm{SiO}$ vapour pressure. We will show that the experimentally determined nucleation rates after correcting for the former strongly overestimated vapour pressure of $\mathrm{SiO}$ are significantly higher than those derived by using the old vapour-pressure data of Schick (1960). Extrapolated to circumstellar conditions, these corrected experimental data predict much higher condensation temperatures than previously.

Additionally we argue that the previous model calculations of circumstellar dust condensation have not properly taken into account that the "dust condensation temperature" derived from analysing infrared (IR) spectra is the temperature of lattice vibrations of the dust particles in the region of onset of massive dust growth in the shell, while the nucleation process is governed by the gas temperature. We show that both temperatures may be significantly different. Both effects cooperate to remove the hitherto suspected strong discrepancy between calculated nucleation temperatures and observed dust temperatures. This again makes clustering of $\mathrm{SiO}$ the hottest candidate for the production of seed particles for silicate dust condensation.

Another way to tackle the problem is to study the chemistry of the gas phase and the kinetics of cluster formation on the basis of reaction kinetics (cf. Reber et al. 2006; Goumans \& Bromley 2012). This is not considered here but will be the subject of a forthcoming paper.

\section{Vapour pressure of SiO}

Experimentally obtained vapour-pressure data for $\mathrm{SiO}$ are discussed in this section, including a brief description of our experimental setup that is based on the Knudsen method (Knudsen 1909). Fundamentally, the mass-loss rate and vapour pressure are measured by a quartz crystal micro balance and the Knudsen cell is heated by electron bombardment with an electron beam evaporator. All experimental results, as well as detailed information regarding the experimental conditions and the samples, are defined in Wetzel et al. (2012).

\subsection{Knudsen method}

The vapour pressure can be determined by measuring the massloss rate of a Knudsen cell, $(\mathrm{d} m / \mathrm{d} t)_{\mathrm{K}}$, at a well-defined temperature $T$. The Knudsen cell is a small container with a small orifice, $A_{\mathrm{O}}$, that is partially filled with an experimental sample. The vapour pressure inside the cell and the mass-loss rate are related by

$P_{\mathrm{K}}(T)=\frac{1}{A_{\mathrm{O}} W_{\mathrm{O}}}\left(\frac{\mathrm{d} m}{\mathrm{~d} t}\right)_{\mathrm{K}} \sqrt{\frac{2 \pi R T}{M}}$,

where $W_{\mathrm{O}}$ is the Clausing factor of the orifice, $R$ the ideal gas constant and $M$ the molecular mass (Margrave 1967). The Clausing factor is the probability that a particle passes the orifice and can be calculated for cylindrical tubes from its geometry (Clausing 1932). The equilibrium vapour pressure can be expressed by the Whitman- Motzfeld equation

$P(T)=\left[1+\frac{A_{\mathrm{O}} W_{\mathrm{O}}}{A}\left(\frac{1}{\alpha(T)}+\frac{1}{W_{\mathrm{Cell}}}-2\right)\right] P_{\mathrm{K}}$,

where $\alpha(T)$ is the vaporization coefficient, $A$ the cross-sectional area of the cell, and $W_{\text {Cell }}$ the Clausing factor of the cell (Whitman 1952; Motzfeldt 1955). Both unknown quantities in Eq. (2), which are the equilibrium vapour pressure and the vaporization coefficient, can be derived from two measurements with different orifice sizes.

\subsection{Experimental setup}

Here we briefly describe our experimental setup (for more details see Wetzel et al. 2012). A basic schematic drawing of the experimental setup is shown in Fig. 1. The quartz crystal microbalance that is placed at a distance $d$ from the Knudsen cell measures the mass changes due to condensing particles during the molecular effusion from the Knudsen cell. The angular dependence of the beam profile can be calculated from the equations given in Clausing (1930) and Dayton (1956) that directly relate the signal detected from the quartz crystal micro-balance to the mass loss of the Knudsen cell. The temperature is determined by a temperature calibration that gives a relation between the temperature in the cell and the heating power controlled during the measurements. The reliability and performance quality of this experimental approach has been verified by a precise reproduction of the vapour pressure of copper (Wetzel et al. 2012). 


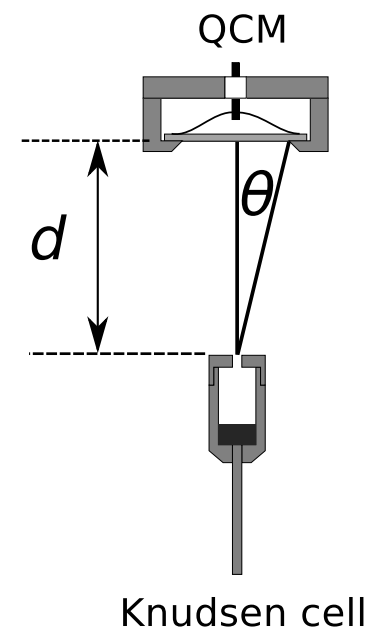

Fig. 1. Schematic drawing of the experimental setup for the Knudsen method. The quartz crystal microbalance (QCM) is placed at a distance $d$ from the Knudsen cell and measures the mass rate of the condensing particles during effusion from the cell.

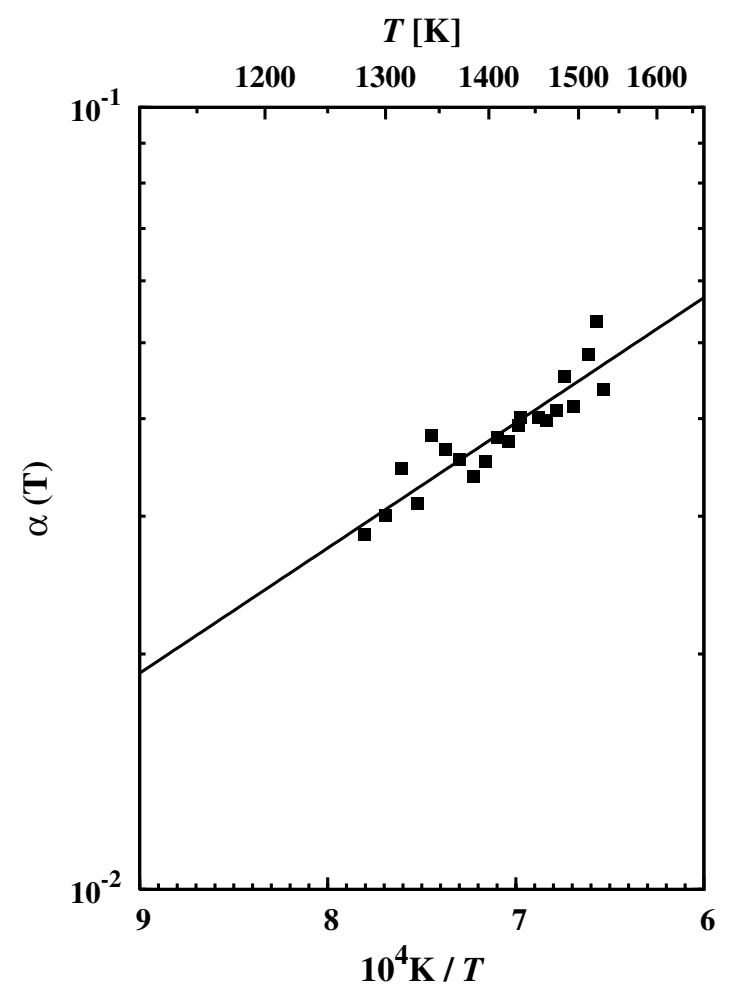

Fig. 2. Vaporization coefficient of solid $\mathrm{SiO}$ derived from the experimental measurements of the mass-loss rate obtained for two orifice diameters of the Knudsen cell. The full line is the approximation according to Eq. (3).

\subsection{Experimental results}

Two orifices with different diameter $(0.50 \mathrm{~mm}$ and $0.79 \mathrm{~mm})$ of the Knudsen cell were employed in order to determine the vapour pressure, as well as the vaporization coefficient of SiO. Figure 2 shows the vaporization coefficient of $\mathrm{SiO}$ derived from the experimental outcomes as a function of temperature. The results can be approximated by an Arrhenius law,

$\alpha(T)=\alpha_{0} \mathrm{e}^{-T_{0} / T}$

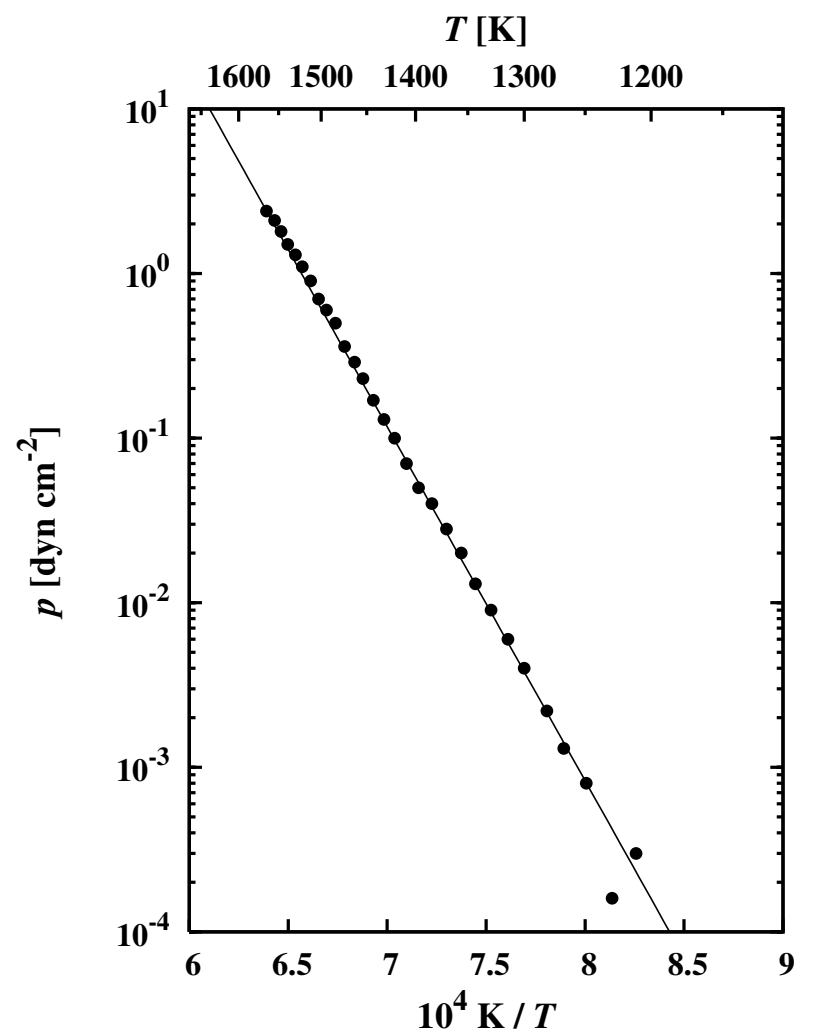

Fig. 3. Equilibrium vapour pressure of solid $\mathrm{SiO}$ derived from the experimental measurements of the mass-loss rate obtained for two orifice diameters of the Knudsen cell (filled circles). The full line is the analytic approximation according to Eq. (4).

with

$\alpha_{0}=0.521 \pm 0.156, \quad T_{0}=3686 \pm 428$

for the temperature range between $1275 \mathrm{~K}$ and $1525 \mathrm{~K}$. This shows that the kinetics of vaporization involves a modest activation energy barrier.

The equilibrium vapour pressure of $\mathrm{SiO}$ over solid silicon monoxide was calculated by using the vaporization coefficient described by Eq. (3). The equilibrium vapour pressure is plotted in Fig. 3 as a function of inverse temperature. From a best fit to the experimental data points, the vapour pressure of $\mathrm{SiO}$ molecule over solid silicon monoxide in units of $\mathrm{dyn} \mathrm{cm}^{-2}$ is approximated by

$P=\exp \left(-\frac{T_{v}}{T}+S_{v}\right)$

with

$T_{v}=49520 \pm 1400 \mathrm{~K}, \quad S_{v}=32.52 \pm 0.97$

\section{Stability limits}

Dust is formed in space in a variety of environments either in a continuous fashion as in stellar winds or brown dwarf atmospheres or as short events in stellar ejecta following explosive events, such as in supernovae or in novae. The elemental composition of dust-forming matter may also be very diverse in different kinds of objects. Here we consider dust formation from oxygen-rich material with a standard cosmic element mixture (cf., e.g., Lodders et al. 2009). The most abundant dust materials 


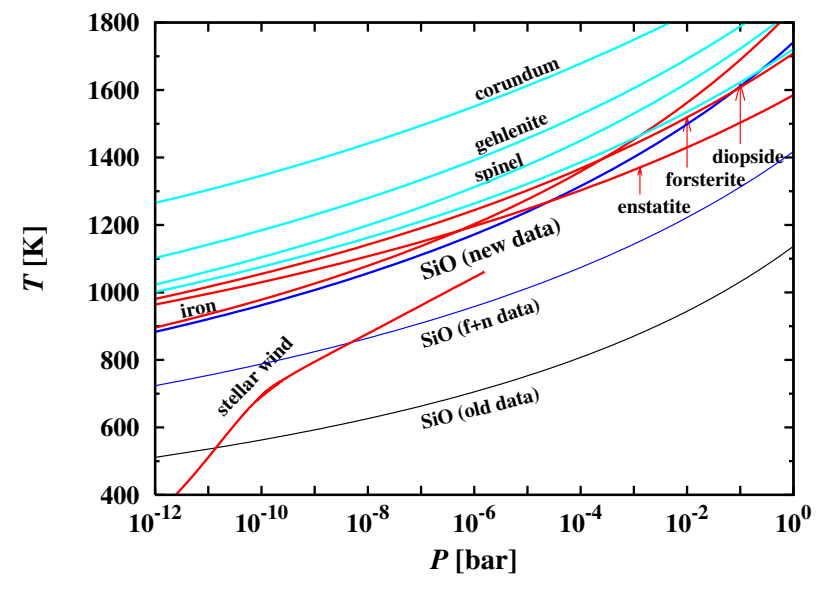

Fig. 4. Stability limit of solid $\mathrm{SiO}$ for the new data from our laboratory measurements for data from Ferguson \& Nuth (2008) and for the old data of Schick (1960). Also shown are stability limits for the most abundant refractory compounds formed from an oxygen-rich element mixture with cosmic standard element abundances. The dashed line corresponds to the $p-T$-trajectory of a dust-driven stellar wind.

that are expected to form from the most abundant refractory elements in such an element mixture, i.e. from $\mathrm{Si}, \mathrm{Mg}$, and $\mathrm{Fe}$, are magnesium-rich silicates and metallic iron (cf., e.g., Gail 2010). From the somewhat less abundant, but even more refractory elements $\mathrm{Al}$ and $\mathrm{Ca}$, the minerals corundum (or hibonite), spinel, gehlenite, and diopside are formed. The upper stability limits or the field of stability in the $p-T$-plane of these compounds are shown in Fig. 4. They are calculated for chemical equilibrium states between the gas phase and condensed phases.

As a new feature, we show the upper stability limit of $\mathrm{SiO}$ according to our new measurements of the vapour pressure of $\mathrm{SiO}$ molecules over solid $\mathrm{SiO}$. This curve in the $p$ - $T$-plane is constructed from the relation

$p \frac{\epsilon_{\mathrm{Si}}}{2+\epsilon_{\mathrm{He}}}=p_{\mathrm{vap}}(T)$.

Here $p$ is the gas pressure, $p_{\text {vap }}$ the vapour pressure of $\mathrm{SiO}$ molecules over solid $\mathrm{SiO}$ according to Eq. (4), and $\epsilon_{\mathrm{Si}}$ and $\epsilon_{\mathrm{He}}$ are the element abundances of $\mathrm{Si}$ and $\mathrm{He}$ with respect to $\mathrm{H}$. The left-hand side corresponds to the pressure of $\mathrm{SiO}$ molecules in the gas phase in an element mixture with cosmic composition. Here we made use of the fact that $\mathrm{H}$ is completely associated to $\mathrm{H}_{2}$ and almost all $\mathrm{Si}$ is bound in $\mathrm{SiO}^{1}$ in the pressure and temperature regime of interest.

For comparison we also show in Fig. 4 the stability limits (i) for solid $\mathrm{SiO}$ resulting from the vapour pressure data reported by Ferguson \& Nuth (2008), i.e. calculated from Eq. (4), but with coefficients

$T_{v}=40850 \pm 1270 \mathrm{~K}, \quad S_{v}=32.93 \pm 0.90$

(in units dyn $\mathrm{cm}^{-2}$ ) and (ii) from the old vapour pressure data for solid SiO of Schick (1960)

$p_{\text {vap }}=\exp \left(26.66-\frac{25670}{T}\right)$

(in units dyn $\mathrm{cm}^{-2}$ ). From Fig. 4 it is immediately obvious that the condensation temperatures following from the data of Schick (1960) are unrealistically low.

\footnotetext{
1 In an oxygen-rich element mixture and at the temperatures of interest most of the $\mathrm{Si}$ is bound in $\mathrm{SiO}$, but some of it is bound in $\mathrm{SiS}$. This, as well as all other Si-bearing compounds, is neglected.
}

For our new data, as well as for the recent data of Ferguson \& Nuth (2008), the stability limit of solid $\mathrm{SiO}$ is still at a lower temperature than the upper stability limits of the more refractory silicates and the $\mathrm{Ca}-\mathrm{Al}$-compounds, but with the strongly reduced vapour pressure determined by our measurements and those of Ferguson \& Nuth (2008), it is not as unfavourably low for condensation under circumstellar conditions as was suggested by the old data of Schick (1960). The strong upward shift of the stability limit according to the new data again makes $\mathrm{SiO}$ a candidate for the seed particle formation in M stars. First steps to investigate this have already performed by Nuth \& Ferguson (2006) and Paquette \& Nuth (2011). Here we follow a different approach.

\section{Application to SiO nucleation}

The details of the reaction mechanisms standing behind the nucleation of $\mathrm{SiO}$ are not yet known, but some experimental results have been obtained by Nuth \& Donn (1982) that give insight into the conditions under which $\mathrm{SiO}$ nucleation occurs. It seems worthwhile to look at how these results change if corrected for the much reduced vapour pressure of $\mathrm{SiO}$.

\subsection{Re-evaluation of the Nuth \& Donn data}

In their laboratory experiment Nuth \& Donn (1982) evaporated solid $\mathrm{SiO}$ from a crucible into a hydrogen atmosphere in order to prepare a gas mixture that only contains $\mathrm{SiO}$ and $\mathrm{H}_{2}$ molecules. In this case, any observed condensation necessarily starts with cluster formation from $\mathrm{SiO}$ molecules ${ }^{2}$.

They determined the temperature and the partial pressure of $\mathrm{SiO}$ at which an onset of smoke formation can be observed. This was quantified by taking the instant at which the extinction of a light beam with wavelength $\lambda=200 \mathrm{~nm}$ traversing the condensation zone reached a level of $1 \%$, as the instant where avalanche nucleation commences. Unfortunately the rate of nucleation $J$ at the onset of detectable smoke formation could only be estimated crudely. It was argued that the value of $J$ at this instant is between $10^{8}$ and $10^{10}$ particles $\mathrm{cm}^{-3} \mathrm{~s}^{-1}$.

The results were presented as a set of values of temperature $T$ and supersaturation ratio

$S=\frac{p_{1}}{p_{\text {vap }}}$

with $p_{1}$ the partial pressure of $\mathrm{SiO}$ in the condensation zone, and $p_{\text {vap }}$ the vapour pressure of $\mathrm{SiO}$ molecules over solid $\mathrm{SiO}$ at temperature $T$. The vapour pressure was calculated by Nuth \& Donn (1982) from the formula given by Schick (1960), Eq. (7). The partial pressure $p_{1}$ or particle densities $n_{1}=p_{1} / k T$ of $\mathrm{SiO}$ molecules in the gas phase in the condensation zone was calculated (i) from the vapour pressure of $\mathrm{SiO}$ in the crucible at the temperature of the crucible and (ii) from the dilution as the vapour diffused away from the crucible to the condensation zone. The pressure in the condensation zone was estimated to be one tenth of the vapour pressure at the crucible.

For a new evaluation of the experimental results of Nuth \& Donn (1982), we read values for $S$ and $T$ from their Fig. 3. We can convert these data for $S$ at given $T$ to a new set of data based on the improved vapour pressure formula for $\mathrm{SiO}$ in the following way: first we calculate a vapour pressure $p_{\text {vap }}$ from the temperature $T$ by means of Eq. (7) and the corresponding pressure

2 For this reason we do not consider the results of Nuth \& Donn (1983) for the more complex $\mathrm{Si}-\mathrm{O}-\mathrm{Mg}$ system. 


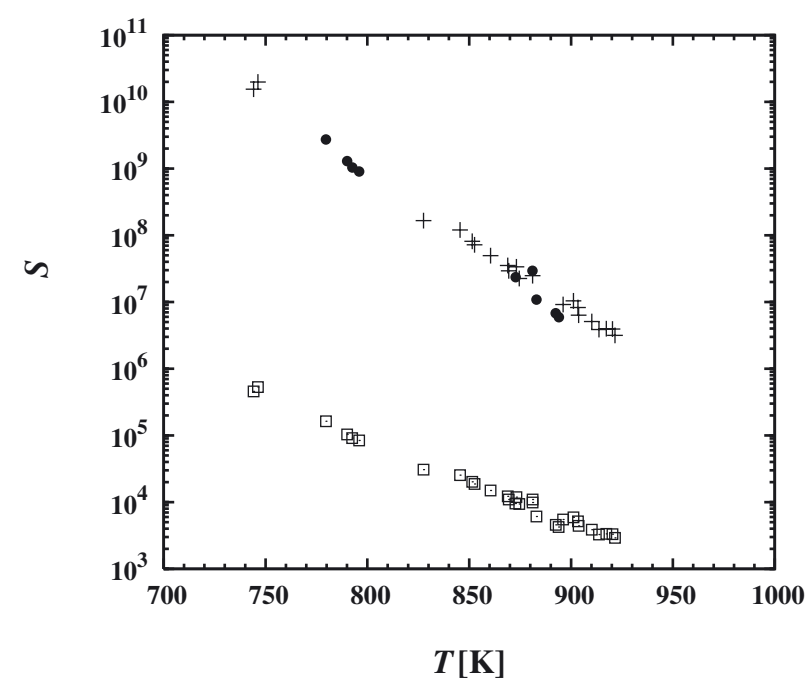

Fig. 5. Supersaturation at onset of avalanche nucleation from the experiment of Nuth \& Donn (1982), re-evaluated with the new vapour pressure data for $\mathrm{SiO}$. Black dots: data for the nucleation experiments conducted at $p_{\mathrm{H}_{2}}=50$ Torr. Crosses: data for experiments conducted at $p_{\mathrm{H}_{2}}=35$ Torr. Open sqares: supersaturation based on old vapour pressure data, as given by Nuth \& Donn (1982), for the same set of experiments.

$p_{1}$ from the value of $S$. Considering that this is assumed to equal one tenth of $p_{\text {vap }}$ calculated with the crucible temperature $T_{\mathrm{c}}$ we reconstruct from Eq. (7) the temperature $T_{\mathrm{c}}$. Then we calculate with this temperature a new value for $p_{1}$ at the crucible by means of Eq. (4) and take one tenth of this as the corrected value for $p_{1}$ in the condensation zone. From the same equation we calculate a new value of $p_{\text {vap }}(T)$ in the condensation zone, and from this and the corrected value of $p_{1}$ a new value for $S$. This defines a new set of values for $S$ at given $T$ that correspond to the fixed value of $J_{\mathrm{av}}=10^{9 \pm 1}$ particles $\mathrm{cm}^{-3} \mathrm{~s}^{-1}$ in the experiments of Nuth \& Donn (1982), but now based on the improved vapour pressure of solid SiO.

The results are shown in Fig. 5 for the two series of measurements with a total pressure of $p_{\mathrm{H}_{2}}=35$ Torr and $p_{\mathrm{H}_{2}}=50$ Torr. This looks very much the same as the corresponding Fig. 3 of Nuth \& Donn (1982), except that the new values for $S$ are more than a factor of a thousand higher than those calculated with the old vapour pressure formula (7).

\subsection{Fit for the nucleation rate}

Next we follow the same procedure as Nuth \& Donn (1982) and check that the results can be fitted with the formula resulting from the Zeldovich theory of homogeneous nucleation (Zeldovich 1943) that gives the following result for the nucleation rate

$J=\left(\frac{2 \sigma}{\pi m}\right)^{\frac{1}{2}} V \alpha n_{1}^{2} \exp \left(-\frac{16 \pi \sigma^{3} V^{2}}{3 k^{3} T^{3}(\ln S)^{2}}\right)$.

Here $\sigma$ is the surface energy of the condensed phase, $V$ the volume of the $\mathrm{SiO}$ molecule in the solid, $A$ and $m$ the atomic weight and mass of the $\mathrm{SiO}$ molecule, $\alpha$ the sticking coefficient at the size of the critical cluster, and $n_{1}=p_{1} / k T$ the particle density of $\mathrm{SiO}$ molecules in the gas phase.

Equation (9) predicts that for a constant nucleation rate $J_{\mathrm{av}}$ as in the measurements of Nuth \& Donn (1982), a linear

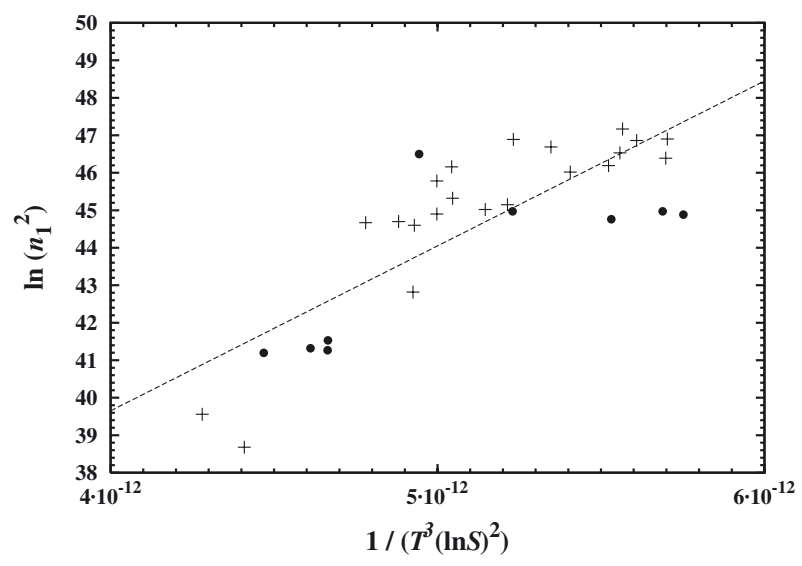

Fig. 6. Plot of $\ln n_{1}^{2}$ versus $\left[T^{3}(\ln S)^{2}\right]^{-1}$. The dashed line shows the best fit of a linear relation, Eq. (10). Symbols as in Fig. 5.

relationship should exist between $\ln n_{1}$ and $T^{3} \ln S$ of the form

$\ln n_{1}^{2}=a \frac{1}{T^{3}(\ln S)^{2}}+b$

with constants $a$ and $b$ given by

$a=\frac{16 \pi \sigma^{3} V^{2}}{3 k^{3}}$
$b=\ln \frac{J_{\mathrm{av}}}{\sqrt{\frac{2 \sigma}{\pi m}} V \alpha}$.

This relationship rests on assumptions that may hold for macroscopic particles but are hard to justify for molecular-sized particles. Nevertheless, one can speculate that the dependency of the classical nucleation rate on the basic macroscopic parameters $T$, $S$, and $n_{1}$ is essentially correct, except that the constants in this equation cannot be derived meaningfully within the framework of a macroscopic theory. This means that we assume that the nucleation rate $J$ is given by

$J=n_{1}^{2} \exp \left(B-\frac{a}{T^{3}(\ln S)^{2}}\right)$

with constants $a$ and

$B=\ln J_{\text {av }}-b$,

to be empirically determined.

One can check this hypothesis by fitting the experimental results of Nuth \& Donn (1982) for $n_{1}$ and $S$ for given $T$ by the linear relation (10). The result is shown in Fig. 6 where $\ln n_{1}^{2}$ is plotted against $\left[T^{3}(\ln S)^{2}\right]^{-1}$ for the experimental data corrected for the new values for the vapour pressure. Figure 6 is completely analogous to Fig. 4 of Nuth \& Donn (1982) except that the data are different. The scatter of the data is rather large and the range of values regrettably rather narrow, but a linear dependency of $\ln n_{1}^{2}$ on $\left[T^{3}(\ln S)^{2}\right]^{-1}$ seems to exist. The least square fitting gave the following results for the coefficients of the linear relation for the corrected data

$a=(4.40 \pm 0.61) \times 10^{12}, \quad b=22.05 \pm 3.14$.

From this, we obtain the following analytical approximation to the experimental data on the nucleation rate by $\mathrm{SiO}$

$J=n_{1}^{2} \exp \left((1.33 \pm 3.1)-\frac{(4.40 \pm 0.61) \times 10^{12}}{T^{3}(\ln S)^{2}}\right)$ 


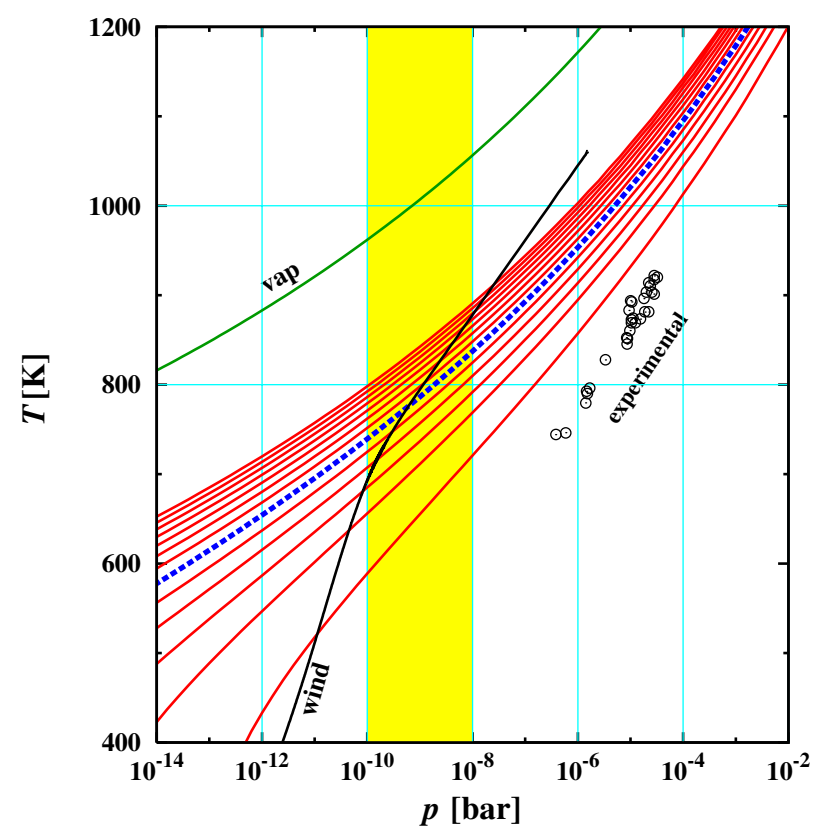

Fig. 7. Lines of constant nucleation rate per H-nucleus according to the numerical fit to the experimental data. The red lines correspond to values of $J / N_{\mathrm{H}}$ from $10^{-34} \mathrm{~s}^{-1}$ (top) to $10^{-10}$ (bottom) in steps of $10^{2}$. The shaded area indicates the range of pressures typically encountered at the inner edge of dust shells. The thick dashed (blue) line corresponds to the critical nucleation rate of $J / N_{\mathrm{H}}=10^{-20}$ particles $\mathrm{cm}^{-3} \mathrm{~s}^{-1}$ where massive dust nucleation commences under circumstellar conditions. The uppermost (green) line corresponds to the stability limit if solid $\mathrm{SiO}$ is in equilibrium with a gas with standard cosmic elemental abundances. The line denoted as "wind" corresponds to the $p-T$-trajectory of a wind model. The circles correspond to the data points of the laboratory experiments, if the pressure of $\mathrm{SiO}$ in the condensation zone is converted into an equivalent pressure of a gas with cosmic elemental composition.

in units $\mathrm{cm}^{-3} \mathrm{~s}^{-1}$. This may be used as a purely empirical fit formula to nucleation rates, the analytical form of which is motivated by theoretical considerations and the constants of which are determined from the results of laboratory experiment.

\subsection{Circumstellar SiO-nucleation}

We assume that the nucleation rate calculated from Eq. (16) remains applicable over a wider range of condensation conditions than that for which the experiments were performed because the coefficients in this expression are essentially determined by molecular and solid phase-properties and not so much by the pressure-temperature conditions of the laboratory experiments. We use this to calculate nucleation rates based on clustering of $\mathrm{SiO}$ molecules for astrophysical problems.

Figure 7 shows lines of constant nucleation rate in the $p$ - $T$-plane per $\mathrm{H}$-atom, $J^{\prime}=J / N_{\mathrm{H}}$, calculated in this way for an oxygen-rich element mixture. Here $N_{\mathrm{H}}$ is the particle density of $\mathrm{H}$ nuclei if all hydrogen atoms are present as free atoms. The particle density of $\mathrm{SiO}$ molecules is assumed to be given by $n_{1}=\epsilon_{\mathrm{Si}} N_{\mathrm{H}}$, and the supersaturation ratio $S$ is calculated by using our fit-formula, Eq. (4), for the vapour pressure of $\mathrm{SiO}$.

If we have a typical number of dust grains of the order of $3 \times$ $10^{-13}$ per hydrogen nucleus and assume that these are formed in about one year, then the formation rate would be about $10^{-20}$ seed particles per $\mathrm{H}$ nucleus per second. This is the black line in Fig. 7, which marks the critical pressure-temperature combination where massive dust particle formation occurs.
For typical pressures between $10^{-10}$ bar at mass-loss rates of $10^{-6} M_{\odot} a^{-1}$ and $10^{-8}$ bar at mass-loss rates of $10^{-4} M_{\odot} a^{-1}$, the critical rate corresponds to temperatures roughly between $720 \mathrm{~K}$ and $850 \mathrm{~K}$. This range of gas temperatures for the onset of massive dust formation is predicted by the empirical nucleation rate (16).

For comparison, Fig. 7 also shows the $p$ - $T$ combinations that correspond to the data points of Nuth \& Donn (1982). Their pressure of $\mathrm{SiO}$ in the condensation zone is converted to the total pressure in an element mixture with cosmic abundances via

$p=\frac{p_{1}}{2 \epsilon_{\mathrm{Si}}}$

with Si abundance $\epsilon_{\mathrm{Si}}$ and neglecting He. This gives an impression of the extrapolation that is required if the results are applied to circumstellar nucleation.

\section{Models for dust-driven winds}

As a simple first application of the new nucleation rate, some models for dust formation in outflows from $\mathrm{M}$ stars are calculated. The aim of this is to check which condensation temperatures of dust result if the nucleation rate for $\mathrm{SiO}$ is applied to circumstellar dust condensation. It is assumed that the condensation of silicate dust is triggered by the nucleation of $\mathrm{SiO}$ clusters from the gas phase and that these seed particles later grow to magnesium-iron silicate dust grains by collecting more $\mathrm{SiO}$ molecules and $\mathrm{Mg}$ and $\mathrm{Fe}$ atoms from the gas phase, while reaction with $\mathrm{H}_{2} \mathrm{O}$ provides the required additional oxygen atoms for building the $\mathrm{SiO}_{4}$ tetrahedrons of the silicate compounds.

In a stellar outflow more than one dust component may be formed in oxygen-rich matter (e.g. Gail \& Sedlmayr 1998a). If some components are already formed at higher temperatures than the silicates (corundum for instance) and if their opacity suffices to drive the outflow velocities to supersonic values, then the gas density has strongly decreased by expansion if the temperature drops below the stability limits of silicates. In that case silicate dust probably precipitates on top of the seed particles formed at higher temperatures, so separate nucleation by $\mathrm{SiO}$ is probably not important. If, however, the subsonic-supersonic transition in the flow is due to silicate dust condensation, then the whole process is governed by the nucleation of silicates. Then we have to deal with dust-driven winds. Here we perform some sample calculations for dust-driven, spherically symmetric and stationary wind models of giant stars. Other possible cases where seed particles with different origins are present will not be considered.

\subsection{Model assumptions}

The wind models including dust formation and growth are calculated by the methods outlined in Gail et al. (1984) and Gail \& Sedlmayr $(1985,1987)$. In those papers, the condensation of graphite dust in $\mathrm{C}$ stars was considered, but the modifications required for calculating silicate dust condensation for $\mathrm{M}$ stars are simple and straightforward. We do not repeat, therefore, a description of the details of the method but only give remarks on a few points that change in the case of $M$ stars.

Since it is assumed that the condensation of silicate dust is triggered by nucleation of $\mathrm{SiO}$ clusters from the gas phase, the nucleation rate is assumed to be given by Eq. (16). The final growth of macroscopic dust grains is assumed to result in the formation of amorphous silicates with an iron-rich composition. That such material is formed in most circumstellar 
Table 1. Models of spherically-symmetric and stationary dust-driven winds of AGB stars with different mass-loss lates, based on the empirical nucleation rate for $\mathrm{SiO}$, Eq. (16).

\begin{tabular}{|c|c|c|c|c|c|c|c|c|c|c|}
\hline \multirow{3}{*}{$\begin{array}{l}\text { Quantity } \\
\text { Mass-loss }\end{array}$} & \multicolumn{7}{|c|}{ Symbol } & \multicolumn{3}{|l|}{ Unit } \\
\hline & \multicolumn{9}{|c|}{ Stellar parameters } & \multirow[b]{2}{*}{$10^{-5} M_{\odot} \mathrm{yr}^{-1}$} \\
\hline & $\dot{M}$ & 1 & 2 & 3 & 5 & 10 & 20 & 30 & 50 & \\
\hline Mass & $M_{*}$ & 1 & 1 & 1 & 1 & 1 & 1 & 1 & 1 & \\
\hline Luminosity & $L_{*}$ & 5 & 5 & 5 & 5 & 5 & 10 & 10 & 10 & $10^{3} L_{\odot}$ \\
\hline Temperature & $T_{*}$ & 2700 & 2700 & 2700 & 2700 & 2700 & 2700 & 2700 & 2700 & \\
\hline Radius & $R_{*}$ & 323 & 323 & 323 & 323 & 323 & 456 & 456 & 456 & $R_{\odot}$ \\
\hline \multicolumn{11}{|c|}{ Sonic point } \\
\hline Position & $r_{\mathrm{c}}$ & 9.21 & 7.98 & 7.50 & 7.05 & 6.60 & 6.42 & 6.20 & 5.93 & $R_{*}$ \\
\hline Temperature & $T\left(r_{\mathrm{c}}\right)$ & 629.1 & 675.9 & 697.1 & 719.0 & 743.2 & 753.4 & 766.9 & 783.7 & $\mathrm{~K}$ \\
\hline Pressure & $p\left(r_{\mathrm{c}}\right)$ & 1.75 & 4.85 & 8.35 & 16.0 & 37.1 & 39.5 & 64.2 & 118 & $10^{-10}$ bar \\
\hline Degree of condensation & $f\left(r_{\mathrm{c}}\right)$ & 13.4 & 14.06 & 14.5 & 15.4 & 17.6 & 6.37 & 6.15 & 6.77 & $\%$ \\
\hline \multicolumn{11}{|c|}{ Dust shell } \\
\hline Terminal velocity & $v_{\infty}$ & 4.42 & 5.42 & 6.08 & 7.01 & 8.67 & 10.71 & 11.81 & 13.10 & $\mathrm{~km} \mathrm{~s}^{-1}$ \\
\hline Terminal condensation & $f_{\infty}$ & 19.4 & 26.9 & 33.9 & 47.2 & 71.4 & 62.7 & 75.8 & 88.3 & $\%$ \\
\hline Rosseland optical depth & $\tau_{\mathrm{R}}$ & 0.36 & 0.75 & 1.03 & 2.37 & 6.21 & 5.26 & 9.31 & 18.02 & \\
\hline Visual optical depth & $\tau_{0.5 \mu \mathrm{m}}$ & 0.97 & 2.01 & 3.26 & 6.39 & 16.75 & 14.19 & 25.10 & 48.60 & \\
\hline
\end{tabular}

dust shells around $\mathrm{M}$ stars is known from analysis of their IR spectra (see Molster et al. 2010; Habing 1996, for reviews). Additionally it has been known for a few years from laboratory analysis of the composition of silicate grains from AGB stars (Vollmer et al. 2009; Nguyen et al. 2010; Bose et al. 2010) that amorphous silicate dust grains from AGB stars have widely varying iron content, where iron-poor grains are rare. This is an important point because the extinction properties of the silicates in the near IR, hence, the possibility of driving a wind by radiation pressure on silicate dust strongly depends on the iron content of the silicates. It has not always been adequately considered in the literature that real amorphous silicate grains are not iron-poor, except for the crystalline component.

A compilation of the equations required for calculating the silicate dust and other dust components in $\mathrm{M}$ stars is given in Ferrarotti \& Gail (2006). Here for simplicity we consider only a single amorphous silicate dust component. Furthermore we assume that this has the composition $\mathrm{MgFeSiO}_{4}$; i.e., we do not include a calculation of the mixing ratio between the two end members of the solid solution in the present model calculation. A composition like the assumed one will be the outcome of nonequilibrium condensation in a rapidly cooling environment because of nearly equal abundances of $\mathrm{Si}, \mathrm{Mg}$, and $\mathrm{Fe}$ in the standard cosmic element mixture (see, e.g., Lodders et al. 2009). Such a composition is also compatible with the results of the laboratory analysis of dust grains from AGB stars, though the observed scatter in composition is large (Bose et al. 2010).

The basic net chemical reaction for the silicate condensation is assumed to be

$\mathrm{SiO}+\mathrm{Mg}+\mathrm{Fe}+3 \mathrm{H}_{2} \mathrm{O} \longrightarrow \mathrm{MgFeSiO}_{4}(\mathrm{~s})+3 \mathrm{H}_{2}$.

For calculating the growth rate of dust grains (see Ferrarotti \& Gail 2006), one needs the abundances of the gas phase species in this reaction equation. The composition of the gas phase is treated in a simple way. In oxygen-rich matter the $\mathrm{Si}$ in the gas phase almost exlusively forms $\mathrm{SiO}$, all $\mathrm{O}$ not bound in $\mathrm{SiO}$ and $\mathrm{CO}$ forms $\mathrm{H}_{2} \mathrm{O}$, while $\mathrm{Mg}$ and $\mathrm{Fe}$ are mainly present as free atoms. We simpy describe this as the gas phase composition and do not explicitly calculate its chemical composition.

The dust opacity is calculated with the complex index of refraction of the dirty silicate model of Ossenkopf et al. (1992, their set 1 ), which is well suited to modelling the IR emission from the dust shells of $\mathrm{M}$ stars. The absorption and scattering efficiencies $C_{\lambda}$ are calculated in the small-particle limit for spherical grains. From analysing the silicate dust grains from AGB stars identified im primitive meteorites, one knows that the large majority of the grains have diameters significantly less than $1 \mu \mathrm{m}$ (Vollmer et al. 2009; Nguyen et al. 2010; Bose et al. 2010), which justifies the use of this approximation from the red to the far IR spectral region, though at the shortward border of the wavelength region where absorption and scattering of stellar photons transmit the momentum to the dust grains (mainly the range $0.5 \lesssim \lambda \lesssim 3 \mu \mathrm{m}$ ), one comes to the limit of validity of this approximation. Investigation of silicate grains from AGB stars (Vollmer et al. 2009; Nguyen et al. 2010) show the grains to be somewhat elongated, though deviations from sphericity seem to be small for most particles. Therefore we assume for simplicity spherical grains for calculating the dust opacities. (In any case, using a distribution of shapes, like CDE, does not significantly change the model.)

For calculating the radiation pressure on the dusty gas one needs the flux average

$\kappa_{H}=\int_{0}^{\infty} \kappa_{\lambda}^{\text {ext }} H_{\lambda} \mathrm{d} \lambda / \int_{0}^{\infty} H_{\lambda} \mathrm{d} \lambda$.

of the mass-extinction coefficient $\kappa_{\lambda}^{\text {ext }}$ for the dust-gas mixture. Here $4 \pi H_{v}$ is the spectral energy flux density of the radiation field. From radiative transfer calculations of circumstellar dust shells we found that this average can be well approximated by (for details see Appendix A)

$\kappa_{H}=\kappa_{\mathrm{P}}^{\mathrm{ext}}\left(T_{*}\right) \mathrm{e}^{-\tau_{*}}+\left[\kappa_{\mathrm{P}}^{\mathrm{ext}}\left(T_{\mathrm{ph}}\right)+(1-f) \kappa_{\mathrm{R}}^{\mathrm{ext}}\left(T_{\mathrm{d}}\right)\right]\left(1-\mathrm{e}^{-\tau_{*}}\right)$

where $\kappa_{\mathrm{P}}^{\text {ext }}$ is the Planck-average, $\kappa_{\mathrm{R}}^{\text {ext }}$ the Rosseland-average of the extinction coefficient, $T_{*}$ the effective temperature of the star, $T_{\mathrm{ph}}$ the dust temperature at the inner edge of the dust shell, $T_{\mathrm{d}}$ the dust temperature in the shell, and $\tau_{*}$ is the optical depth calculated from the inner edge of the dust shell outwards using $\kappa_{\mathrm{P}}^{\mathrm{ext}}\left(T_{*}\right)$ as opacity. The Eddington factor $f$ can be taken for example from the approxiation of Lucy (1976) (see also Gail \& Sedlmayr 1987, for details). 

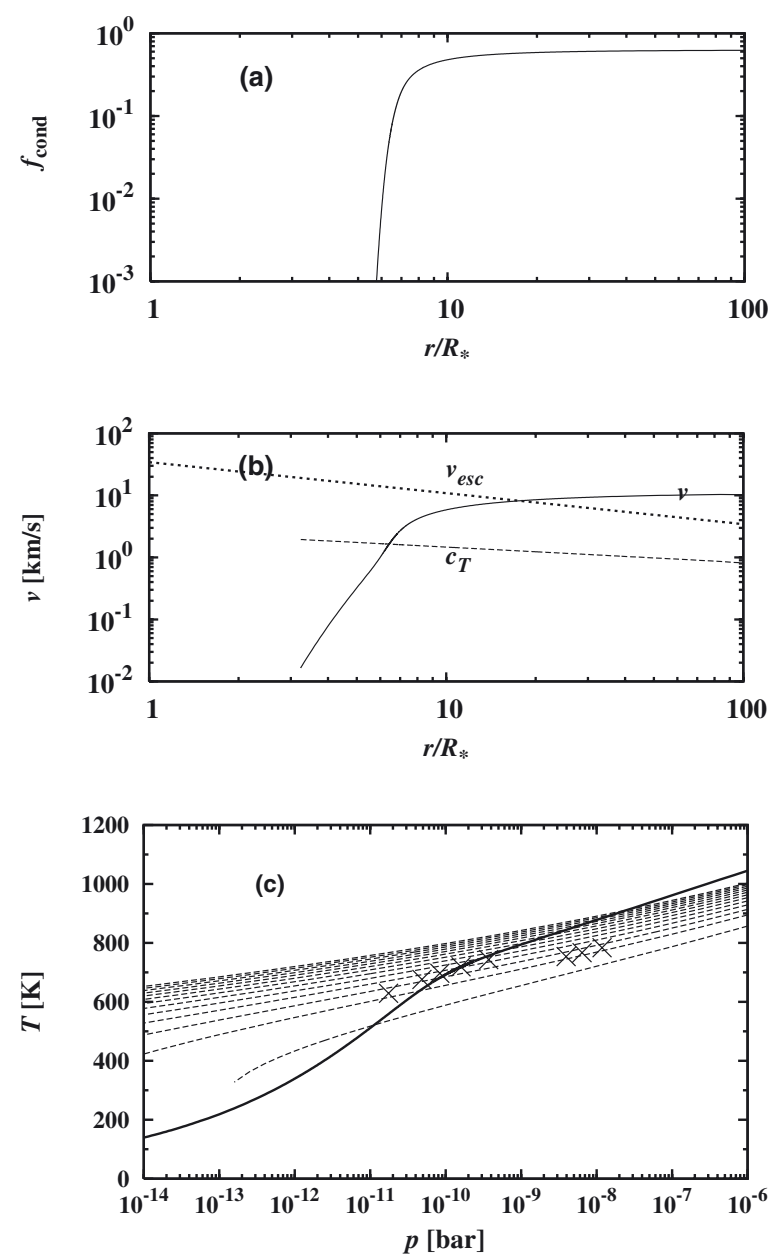

Fig. 8. Some results for a wind model with $\dot{M}=5 \times 10^{-6} M_{\odot} \mathrm{yr}^{-1}$. a) Variation in the degree of condensation of $\mathrm{Si}$ into silicate dust with radial distance from the mass centre. b) Radial variation with distance of outflow velocity $v$ (solid line), sonic velocity $c_{\mathrm{T}}$ (dashed line), and escape velocity from star (dotted line). c) Variation in $p$ and $T$ along the wind (solid line) and lines of constant nucleation rate (dashed lines). The crosses denote the pressure and temperature at the inner edge of the dust shell for the models of Table 1 .

\subsection{Model calculations}

With these assumptions some models are calculated for dustdriven winds of oxygen-rich AGB stars. The assumption of stationarity can hardly be justfied for AGB stars because they are variables. The outerflowing gas is subject to shock waves triggered by the pulsation of the underlying star and the whole structure is far from being stationary. One can only speculate that stationary models describe long-term average properties of such outflows, which is justified, at least in part, by the success of radiative transfer models based on $r^{-n}$ density distributions. These can be used to model IR spectra from dust shells. In any case, the purpose of our present calculation is to see whether the new empirical nucleation rate results in condensation temperatures of silicate dust that mainly agree with what one derives from analysis of IR spectra.

Models are calculated for the higher end $(\dot{M} \gtrsim 1 \times$ $\left.10^{-6} M_{\odot} \mathrm{yr}^{-1}\right)$ of the observed range of mass-loss rates for such stars $\left(\dot{M} \gtrsim 1 \times 10^{-8} M_{\odot} \mathrm{yr}^{-1}\right)$ because the present model does not consider the drift of dust grains relative to the gas. This is not important for higher mass-loss rates because drift velocities are less than the thermal verlocity of the growth species in this case, such that the collision rate of dust grains with growth species is not significantly increased by drift. For low mass-loss rates, however, particle drift dramatically enhances dust growth and so must be considered.

Table 1 shows the stellar parameters chosen for the calculations and some results. Figure 8 shows some details of the model with a mass-loss rate of $\dot{M}=5 \times 10^{-6} M_{\odot} \mathrm{yr}^{-1}$. Its general structure is representative of all our models. One feature of interest is the rather strong increase in the degree of condensation (Fig. 8a), which means that dust appears rather suddenly, and the dust shell has a reasonably well-defined inner boundary. The wind trajectory shown in Fig. 8c shows how an outflowing gas parcel first cools and reduces pressure by expansion, then intrudes into the region of the increasing nucleation of seed particles for silicate dust growth, upon which the resulting strong acceleration results in a rapid blow-out of the gas parcel into low-pressure regions where, finally, growth ceases because of strong gas expansion.

Of particular interest in our context are the typical temperatures at the onset of condensation, because this defines the hottest temperatures of dust grains that may contribute to the IR spectrum from the dust shell. We take the position of the sonic point, where the wind velocity $v$ equals the sonic velocity $c_{\mathrm{T}}$ (see Fig. 8b) as the position of the inner boundary of the dust shell. The distance of this point is given in Table 1 and also the temperature at this radius. At this point substantial condensation has already occurred in order that radiation pressure on the dust component increases to the level that it locally surmounts the gravitational pull by the star. Additional substantial condensation, however, is requiered to enhance radiation pressure for ultimately lifting the matter out of the deep gravitational potential well of the star (see Fig. 8b). The degree of condensation at the sonic point and the final degree of condensation far in the outflow are shown in Table 1.

The table also shows the pressure and temperature at the inner boundary of the dust shell for the models. This outlines the typical range of "condensation temperatures" where avalanche nucleation and dust growth have just begun. The dust grains at the inner border of the dust shell are the hottest dust grains that exist for the considered dust species because of their proximity to the star. Their temperature may be derived from observations of IR spectra, and this temperature can in turn be compared with the theoretical results following from our approximation for the nucleation rate.

\section{Comparison with observations}

We do not present radiative transfer models for dust shells but use data from the literature for dust temperatures at the inner edges of dust shells. In such models of dust shells, it is usually assumed that (i) the dust is formed at some condensation temperature $T_{\mathrm{c}}$ in the outflow; and (ii) the density distribution of dust outwards from this point is proportional to $r^{-2}$. The mean intensity, $J_{v}$, is then calculated from a solution of the radiative transfer problem with the inner boundary condition that we have a star of given luminosity $L_{*}$ and effective temperature $T_{*}$. The dust opacity is calculated from optical constants of materials thought to be analogues of circumstellar dust materials. For M-stars different kinds of silicates and sometimes Al-Ca-bearing minerals are considered in the calculations. The run of temperature in the shell and its inner radius are determined self-consistently from radiative equilibrium between emission and absorption by the dust grains and the solution of the radiative transfer problem.

The kind of dust material used for the modelling, the temperature $T_{\mathrm{c}}$, the corresponding inner radius $R_{\mathrm{c}}$, and the dust density 


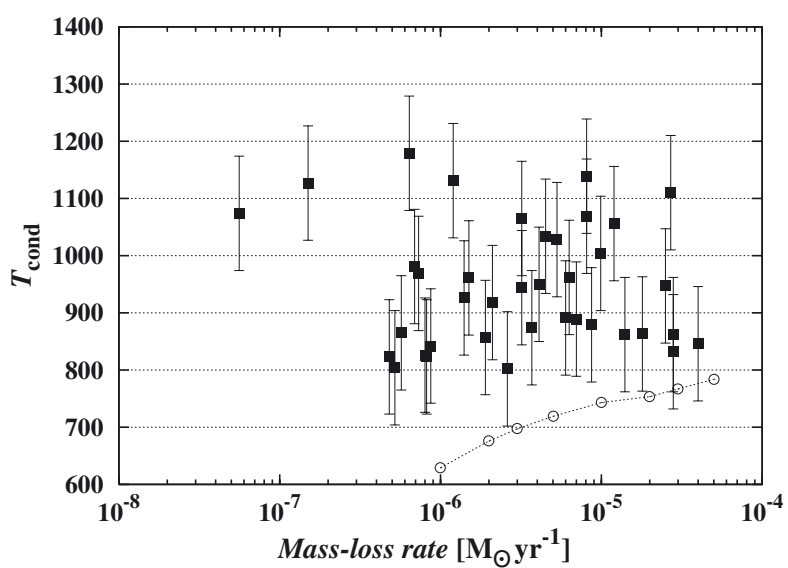

Fig. 9. Temperature at the inner edge of dust shells of AGB stars in the Small and Large Magellanic clouds with estimated errors of the temperature determination (data from Groenewegen et al. 2009). This corresponds to the temperature where massive silicate dust condensation commences in the outflow. The data are plotted against the derived mass-loss rate. Dotted line with open circles denotes the temperature at the sonic point of the models from Table 1.

at $R_{\mathrm{c}}$ are then varied to obtain an optimum fit with observed IR spectra from circumstellar shells. If the outflow velocity is known from, say, the width of CO lines, one can determine a dust mass-loss rate from the dust density and a total mass-loss rate with an assumed gas/dust ratio.

It is generally assumed that the temperature $T_{\mathrm{c}}$ derived this way reflects the dust temperature at the inner edge of the dust shell, where efficient dust formation commences. For this reason, this temperature is called the condensation temperature of the dust. It is to be observed that this temperature corresponds to the lattice temperature in radiative equilibrium of particles, which have grown to such a size that their extinction properties have changed to solid-like behaviour. This does not imply any information on the gas temperature in that region.

From the numerous such studies published in the literature we consider here a set of data for late-type M-stars on the AGB from the Small and Large Magellanic Clouds derived by Groenewegen et al. (2009), because this is a very homogeneous data set both with respect to the acquisition of spectra and the model data derived for all stars with the same method and the same set of opacity data. The condensation temperatures $T_{\mathrm{c}}$ are shown in Fig. 9. Here only data for those objects are used for which the dust temperature at the inner edge could uniquely be determined by the model fit. The figure suggests that there are two different groups of objects with different condensation temperatures. For one group, the typical temperatures are around $880 \mathrm{~K} \pm 100 \mathrm{~K}$. These are the "condensation temperatures" of silicate dust that have to be explained by theoretical calculations of dust formation.

Then there seems to be a second group of objects with condensation temperatures between $1100 \mathrm{~K}$ and $1200 \mathrm{~K}$. They could correspond to objects with an observable production of Ca-Albearing dust species which are thermally more stable than silicates. We neglect such objects because they may represent physically distinct objects.

Figure 9 shows the temperature at the inner inner edge of dust shells in our theoretical models, based on the approximation for the nucleation rate from Sect. 4. At first glance the theoretical condensation temperatures seem to fall short of what is followed from radiative transfer models for observed dust shells by about 100 to $200 \mathrm{~K}$. This cannot result from the big uncertainties

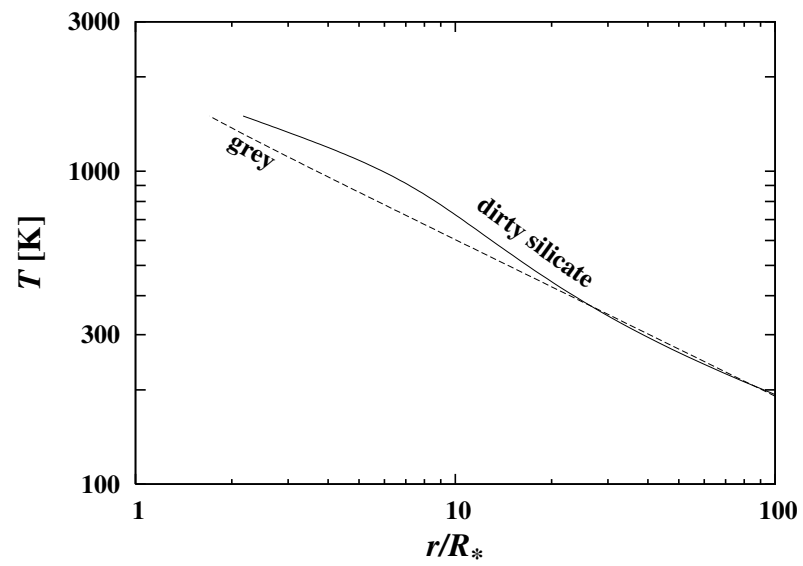

Fig. 10. Dust temperature and grey temperature in optically thin dust shells for a star radiating like a black body with temperature $T_{\text {eff }}=$ $2700 \mathrm{~K}$, calculated for silicate dust with opacities from Ossenkopf et al. (1992).

connected with the conversion of the experimental data of Nuth \& Donn (1982) into the apprimate formula (16) because the nucleation rate depends very steeply on temperature, as can be seen in Fig. 7. Variations in $J$ even over many decades correspond to relativly small temperature changes. The discepancy is too big to result from such errors.

A more likely origin for the difference in condensation temperature is the approximation that the model does not distinguish between the gas temperature that rules nucleation and dust temperature which rules the emission of IR radiation by dust. Generally, both temperatures are significantly different, in particular if dust shells, are optically thin $\left(\tau_{\mathrm{R}} \lesssim 1\right)$. For optically thin to moderately optically thick dust shells, the local mean radiation intensity is dominated by the stellar contribution. The dust temperature in radiative equilibrium is then given by the well known relation

$T_{\mathrm{d}}=W^{\frac{1}{4}} T_{*}\left(\frac{\kappa_{\mathrm{P}}\left(T_{\mathrm{eff}}\right)}{\kappa_{\mathrm{P}}\left(T_{\mathrm{d}}\right)}\right)^{\frac{1}{4}}>W^{\frac{1}{4}} T_{*}=T_{\text {grey }}$,

with the geometrical diluton factor

$W=\frac{1}{2}\left(1-\sqrt{1-\frac{R_{*}^{2}}{r^{2}}}\right)$.

Here $R_{*}$ is the stellar radius, $r$ the radial distance to the stellar centre, $T_{*}$ the effective temperature of the star, and $T_{\text {grey }}$ the temperature of a grey absorber. The variation in the Planck-average

$\kappa_{\mathrm{P}}(T)=\int_{0}^{\infty} \kappa_{v} B_{v}(T) \mathrm{d} v / \int_{0}^{\infty} B_{v}(T) \mathrm{d} v$.

of the dust absorption coefficient with temperature has a significant influence on the dust temperature.

Figure 10 shows the dust temperature of silicate dust material in radiative equilibrium at different distances from a central star with effective temperature $T_{\text {eff }}=2700 \mathrm{~K}$, determined according to Eq. (20), using optical data from Ossenkopf et al. (1992). Additionally, the temperature of a material with grey absorption is shown, for which the monochromatic absorption is independent of wavelength. For silicates the dust temperature is higher than for a grey body because their Planck-averaged absorption coefficient is higher for the effective temperature of the stellar radiation field than for the much lower dust temperatures. Since 
the gas temperature tends to be close to the grey temperature except behind shocks (cf. Schirrmacher et al. 2003), this behaviour can easely explain the remaining difference between the condensation temperature in our models and the observed condensation temperatures. As a result, the laboratory determined nucleation rate for $\mathrm{SiO}$ (Nuth \& Donn 1982), corrected for the the strong reduction of vapour pressure of $\mathrm{SiO}$ required by recent measurements (Wetzel et al. 2012; Nuth \& Ferguson 2006), seems to be able to explain the dust condensation in circumstellar dust shells of AGB stars.

Unfortunately, we presently cannot definitely fix the difference between gas and dust temperature unless the model calculation exlicitly considers a different gas and dust temperature. These more elaborate model calculations will be published elsewhere.

\section{Concluding remarks}

We have re-analysed the long-standing problem of nucleation of silicate dust grains in circumstellar dust shells around oxygenrich evolved stars. Though it has already been proposed at an early stage that this is due to clustering of the abundant $\mathrm{SiO}$ molecules (Donn et al. 1981; Nuth \& Donn 1982), serious problems remained with this hypothesis. The nucleation process was studied by dedicated laboratory expriments by Nuth \& Donn $(1982,1983)$, but the results, if applied to ciurcumstellar conditions, seemed to predict condensation temperatures that are much too low (Gail \& Sedlmayr 1986) compared to the dust temperatures at the inner edge of dust shells derived from IR spectra.

New determinations of the vapour pressure of $\mathrm{SiO}$ by Nuth \& Ferguson (2006) and Ferguson \& Nuth (2008) and that are reported in this paper (see also Wetzel et al. 2012) show that former determinations were grossly in error. Our new measurements yield vapour pressures that are even somewhat lower than obtained by Ferguson \& Nuth (2008).

Nuth \& Ferguson (2006) have already shown that the case for $\mathrm{SiO}$ nucleation may be not as unfavourable as was thought in the past. They use their vapour pressure data to calculate more realistic supersaturation values $S$ and using the approximation given in Nuth \& Donn (1982) they determine the nucleation rate $J$. They showed that condensation temperatures were increased by a lot if the new vapour pressure data are used. But still a significant gap to observed values remains which they try to explain by non-LTE effects in the population of energy levels in $\mathrm{SiO}$ (cf. also Nuth \& Donn 1981).

Here we completely re-evaluated the experimentally determined data for the $\mathrm{SiO}$ nucleation of Nuth \& Donn (1982) with our new vapour-pressure data and derived a fit-formula for the nucleation rate. This can be used in model calculations for stellar outflows. Some explorative calculations were done by calculating stationary dust-driven wind models in a simple approximation. The resulting temperatures at the inner edge of the dust shells were compared to data for the dust condensation temperature derived from analysing IR spectra. It was found that model results based on the new approximation for the nucleation rate are now close to what one observes. We argue that the remaining small gap is due to the difference between gas temperature (responsible for nucleation) and dust temperature (responsible for IR emission), which was not considered in our simple models.

Our result suggests that, indeed, in circumstellar dust shells around M stars silicate dust formation may start with nucleation of SiO. This holds only for those cases where not some different kind of seed particles, which formed well before $\mathrm{SiO}$ nucleation becomes possible (e.g. corundum grains), served as growth centres for the silicate grains. Observed condensation temperatures suggest that both cases seem to occur in circumstellar shells.

The problem of silicate nucleation can, however, not be considered as completely solved, because our model calculations assumed stationary outflows, which are at best a rather crude approximation for winds of AGB stars, because all such stars are variables. Time-dependent model calculations accounting for the shock structure in the winds and accounting for the difference between gas and dust temperature are required before definite conclusions can be draw.

Acknowledgements. This work was supported in part by Forschergruppe 759' and special research progamme SPP 1385 , which both are financed by the "Deutsche Forschungsgemeinschaft (DFG)".

\section{References}

Ali, A., \& Castleman, Jr., A. W. 2005, in IAU Symp., 235, 107 Bose, M., Floss, C., \& Stadermann, F. J. 2010, ApJ, 714, 1624

Clausing, P. 1930, Z. Phys., 66, 471

Clausing, P. 1932, Ann. Phys., 404, 961

Dayton, B. B. 1956, Vacuum Symposium Transactions, 5

Donn, B., Hecht, J., Khanna, R., et al. 1981, Surface Science, 106, 576

Ferguson, F. T., \& Nuth, III, J. A. 2008, J. Chem. Eng. Data, 53, 2824

Ferrarotti, A. S., \& Gail, H.-P. 2006, A\&A, 447, 553

Gail, H.-P. 2010, in Astromineralogy, 2nd edn., ed. T. Hennig (Heidelberg: Springer), 55

Gail, H.-P., \& Sedlmayr, E. 1985, A\&A, 148, 183

Gail, H.-P., \& Sedlmayr, E. 1986, A\&A, 166, 225

Gail, H.-P., \& Sedlmayr, E. 1987, A\&A, 171, 197

Gail, H.-P., \& Sedlmayr, E. 1998a, in The Molecular Astrophysics of Stars and Galaxies, eds. T. W. Hartquist, \& D. A. Williams (Oxford: Oxford University Press), 285

Gail, H.-P., \& Sedlmayr, E. 1998b, Faraday Discussion, 109, 303

Gail, H.-P., Keller, R., \& Sedlmayr, E. 1984, A\&A, 133, 320

Goumans, T. P. M., \& Bromley, S. T. 2012, MNRAS, 420, 3344

Groenewegen, M. A. T., Sloan, G. C., Soszyński, I., \& Petersen, E. A. 2009, A\&A, 506, 1277

Habing, H. J. 1996, A\&ARv, 7, 97

Jeong, K. S., Winters, J. M., Fleischer, A. J., \& Sedlmayr, E. 1998, in A Half Century of Stellar Pulsation Interpretation, eds. P. A. Bradley, \& J. A. Guzik, ASP Conf. Ser., 135, 335

Knudsen, M. 1909, Ann. Phys., 333, 75

Lodders, K., Palme, H., \& Gail, H.-P. 2009, in Landolt-Börnstein, New Series, Group IV, ed. J. E. Trümper (Berlin: Springer), 4, 560

Lucy, L. B. 1976, ApJ, 205, 482

Margrave, J. L. 1967, The Characterization of High-Temperature Vapours (John Wiley and Sons)

Molster, F. J., Waters, L. B. F. M., \& Kemper, F. 2010, in Lect. Notes Phys. (Berlin: Springer Verlag), ed. T. Henning, 815, 143

Motzfeldt, K. 1955, J. Phys. Chem., 59, 139

Nguyen, A. N., Nittler, L. R., Stadermann, F. J., Stroud, R. M., \& Alexander, M. O. 2010, ApJ, 719, 166

Nuth, J. A., \& Donn, B. 1981, ApJ, 247, 925

Nuth, J. A., \& Donn, B. 1983, J. Chem. Phys., 78, 1618

Nuth, J. A. III, \& Donn, B. 1982, J. Chem. Phys., 77, 2639

Nuth, J. A. III, \& Ferguson, F. T. 2006, ApJ, 649, 1178

Ossenkopf, V., Henning, T., \& Mathis, J. S. 1992, A\&A, 261, 567

Paquette, J. A., \& Nuth, III, J. A. 2011, ApJ, 737, L6

Paquette, J. A., Ferguson, F. T., \& Nuth, III, J. A. 2011, ApJ, 732, 62

Reber, A. C., Clayborne, P. A., Reveles, J. U., et al. 2006, Nano Lett., 6, 1190

Schick, H. L. 1960, Chem. Rev., 60, 331

Schirrmacher, V., Woitke, P., \& Sedlmayr, E. 2003, A\&A, 404, 267

Vollmer, C., Brenker, F. E., Hoppe, P., \& Stroud, R. M. 2009, ApJ, 700, 774

Wetzel, S., Pucci, A., \& Gail, H.-P. 2012, J. Chem. Eng. Data, 57, 1594

Wetzel, S., Klevenz, M., Gail, H.-P., Pucci, A., \& Trieloff, M. 2013, A\&A, 553, A92

Whitman, C. I. 1952, J. Chem. Phys., 20, 161

Zeldovich, Y. B. 1943, Acta Physicochim., 18, 1

Page 11 is available in the electronic edition of the journal at http://www . aanda. org 


\section{Appendix A: Approximation for flux-averaged extinction}

In a study of radiative transfer models in circumstellar dust shells (Gail et al., in prep.), it was found that the flux-averaged extinction coefficient can be approximated rather well by the expression given in Eq. (19). This approximation is based on the following considerations.

1. At the inner edge of the dust shell the radiation field is dominated by stellar radiation. The spectral distribution of the flux, $4 \pi H_{\lambda}$, can be approximated by a black body with the effective temperature of the star. The flux-averaged extinction coefficient can be approximated by the Planck-averaged extinction coefficient $\kappa_{\mathrm{P}}^{\text {ext }}\left(T_{*}\right)$ in this case.

2. Moving from the inside into the shell, the stellar contribution to the radiation field diminishes as $\exp \left(-\tau_{*}\right)$, where $\tau_{*}$ is the optical depth calculated from the inner edge with $\kappa_{\mathrm{P}}^{\mathrm{ext}}\left(T_{*}\right)$. At the same time, the contribution of the local dust emission to the total flux increases proportional to $1-\exp \left(-\tau_{*}\right)$.

3. As long as the optical depth of the shell is not very high, the spectral flux distribution from dust emission can be approximated by a black body radiation field with temperature corresponding to the inner edge of the dust shell, $T_{\mathrm{ph}}$. This results in a contribution $\kappa_{\mathrm{P}}^{\mathrm{ext}}\left(T_{\mathrm{ph}}\right)$ to the flux-averaged extinction coefficient.

4. If the shell becomes very thick optically, the flux-averaged extinction coefficient should approach the Rosseland mean of the local radiation field, $\kappa_{\mathrm{R}}^{\mathrm{ext}}\left(T_{\mathrm{d}}\right)$. The approach to the optically thick case can be modelled by the factor $1-f$ with the Eddington factor $f$, which is $f \approx 1$ in an optically thin shell, and approaches $f \approx 1 / 3$ for optically thick shells.

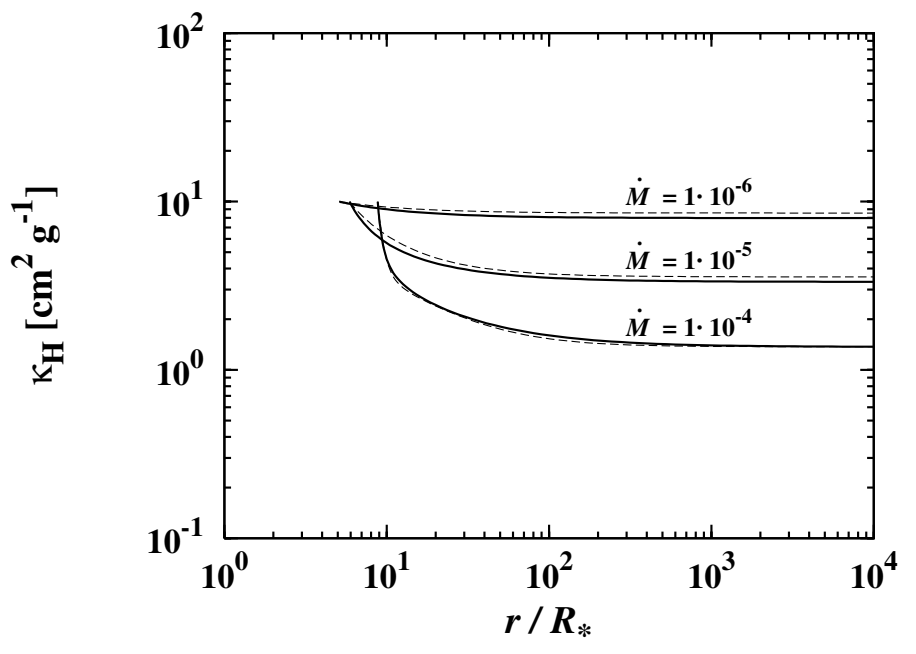

Fig. A.1. Approximation of the flux-averaged extinction coefficient $\kappa_{H}$ in models for a dust shell with a powerlaw wavelength variation of the extinction coefficient for different mass-loss rates (in units $M_{\odot} a^{-1}$ ). Solid line: Result of a complete model calculation of radiative transfer. Dashed line: approximation according to Eq. (19).

As a demonstration of the accuracy of the approximation, Fig. A. 1 compares the result for $\kappa_{H}$ based on a full calculation of radiative transfer, as outlined in Wetzel et al. (2013), using a powerlaw variation $\kappa_{\lambda} \propto \lambda^{-1}$ as a simple model case. An inspection of the figure shows that the approximation for $\kappa_{H}$ is fairly accurate. 